\title{
Síntese de materiais mesoporosos: Um estudo comparativo entre o SBA-15 e o KIT-6
}

\author{
Fernando Rodrigo Dantas Fernandes* (Mestrando do PPGCN na Universidade do Estado do Rio Grande do \\ Norte - UERN);
}

Anne Gabriella Dias Santos (Prof. a do DQ e do PPGCN na Universidade do Estado do Rio Grande do Norte - UERN);

Vinícius Patrício da Silva Caldeira (Prof. do DQ e do PPGCN na Universidade do Estado do Rio Grande do Norte - UERN);

Luiz Di Souza (Prof. a do DQ e do PPGCN na Universidade do Estado do Rio Grande do Norte - UERN;

* Email:fe.rodrigo@hotmail.com

\section{Resumo:}

Materiais mesoporos ordenados têm recebido muita atenção devido às suas potenciais aplicações em catálise, adsorção, troca iônica e fabricação de materiais funcionais avançados. Dentre os materiais mesoporosos o SBA-15 e o KIT- 6, que possuem estruturas hexagonal e cúbica, respectivamente, possuem características interessantes, tais como: alta estabilidade térmica e hidrotérmica, sistemas de poros bastante ordenados, área superficial elevada e elevados diâmetro e volume médio de poros. A síntese desses materiais ocorre de forma hidrotérmica em meio ácido, utilizando o P123 como direcionador de estrutura e o TEOS como fonte de sílica, e na síntese do KIT-6, diferente do SBA-15, ocorre também a adição do Butanol como co-solvente e co-direcionador. O objetivo do presente trabalho é sintetizar e caracterizar os materiais mesoporosos SBA-15 e KIT-6 a fim de se realizar um estudo comparativo entre os materiais, avaliando aspectos estruturais e morfológicos dos mesmos. Os materiais foram sintetizados segundo procedimentos encontrados na literatura e caracterizados através das técnicas de DRX, TG/DTG, Adsorção/Dessorção de N2 e MEV. Os materiais foram obtidos com êxito, apresentando características típicas. Pôde ser observado através das caracterizações as principais diferenças estruturais e morfológicas existentes entre os dois tipos de materiais. O SBA-15 apresenta uma estrutura hexagonal mais fácil de se formar em comparação com a estrutura cúbica do KIT-6. Necessita-se da adição do Butanol na parte inicial da síntese do KIT-6, para que as micelas a se organizem das micelas na forma cúbica.

Pallavras-chave:

Síntese; Caracterização; SBA-15; KIT-6.

Espaço reservado para organização do congresso. 


\section{I ntrodução}

Materiais mesoporos ordenados têm recebido muita atenção devido às suas potenciais aplicações em catálise, adsorção, troca iônica e fabricação de materiais funcionais avançados (PRABHU et al., 2009). Segundo designação da IUPAC, esses materiais possuem diâmetro médio de poros entre 2 e $50 \mathrm{~nm}$. Dentre os materiais mesoporosos o SBA-15 e o KIT- 6, que possuem estruturas hexagonal e cúbica, respectivamente (KO; SHIN; CHOI, 2011; BOULAOUED et al., 2012) tem sido alvo de vários estudos recentemente, devido a suas características promissoras e suas aplicações variadas na área de catálise e meio ambiente (SONI et al., 2009; QIAN et al., 2012; HUSSAIN et al., 2013).

O SBA- 15 foi desenvolvido e sintetizado pela primeira vez na Universidade da Califórnia Santa Bárbara, EUA. Este material apresenta- se como um dos materiais mais investigados e empregados nos últimos tempos (NAKAZAWA et al., 2012), possui uma estrutura mesoporosa unidirecional, elevado diâmetro médio de poros e grande estabilidade térmica e hidrotérmica (ZHAO, et al. 1998a, b). Os seus mesoporos, ordenados hexagonalmente e interligados por microporos, possibilitam o alojamento ou a difusão de grandes moléculas, o que confere a ele uma vasta aplicabilidade na área de catálise (DAO et al., 2008) e adsorção (HIYOSHI et al., 2005)

O material do tipo KIT- 6 é assim denominado por que sua descoberta ocorreu no Instituto Avançado de Ciência e Tecnologia da Corea (Korea Advanced Institute of Science and Technology) (KALBASI; MOSADDEGH, 2012). Esse material apresenta largo diâmetro médio de poro, espessura de parede por volta de 4-6 nm, estrutura simétrica cúbica tridimensional Ia3d (BOULAOUED et al., 2012), grande volume de poro, área superficial elevada, e rede interpenetrante bicontínua de canais (DOU et al., 2011). O KIT-6 possui estrutura semelhante à encontrada no MCM- 48, porém apresenta algumas vantagens: microporos complementares com cerca de $1,8 \mathrm{~nm}$ que interligam os mesoporos (QIAN et al., 2012), maior estabilidade hidrotérmica (FALAHATI et al., 2011) e maior diâmetro de poro (KUMARESAN et al., 2010), o que confere ao material maior aplicabilidade. A estrutura em 3D do KIT-6 e a sua resistência ao bloqueio dos seus poros (KARTHIKEYAN; PANDURANGAN, 2012), o torna um excelente candidato a adsorção e atividade catalítica.

A síntese hidrotérmica desses materiais ocorre em meio ácido, utilizando ácido clorídrico (HCl), sendo a concentração de HCl reduzida na síntese do KIT-6 em comparação ao SBA-15 (FALAHATI et al., 2011). As sínteses do SBA-15 e do KIT-6, foram relatadas pela primeira vez por Zhao et al. (1998a) e Kleitz et al. (2003), respectivamente. Na síntese típica desses materiais utiliza-se basicamente o copolímero tribloco P123 como direcionador de estrutura e o tetraetilortosilicato (TEOS) como fonte de sílica. A diferença principal entre as sínteses desses materiais é o uso do Butanol na síntese do KIT-6, esse reagente é utilizado na etapa inicial da síntese como um co-direcionador e co-solvente (WANG et al.,2009).

Devido esses materiais se apresentarem muito importante dentro da sua classe (materiais mesoporosos) e mesmo com sínteses semelhantes apresentam algumas diferenças no procedimento, o que modifica totalmente as suas características estruturais, o presente trabalho tem como objetivo sintetizar e caracterizar os materiais mesoporosos SBA-15 e KIT-6 a fim de fazer um estudo comparativo e avaliar as suas características estruturais e morfológicas.

\section{Material E Métodos}

\subsection{Síntese do SBA-15}

A amostra de SBA-15 foi sintetizada pelo método hidrotérmico, proposto por Zhao et al. (1998a), usando o copolímero tribloco P123 (EO20PO70EO20), ácido clorídrico ( $\mathrm{HCl}$ ) 37\%, água destilada e o tetraetilortosilicato (TEOS) como fonte de sílica. O procedimento consistiu na dissolução do P123 em água e $\mathrm{HCl}$ por 2 horas em uma temperatura que variou de 35 a $40^{\circ} \mathrm{C}$, logo após, adicionou- se a fonte de sílica e deixou a mistura sob agitação a mesma temperatura por 24 horas. Em seguida colocou- se o gel de síntese em uma autoclave de teflon revestida de aço inoxidável e colocou- se na estufa por 48 horas a $100{ }^{\circ} \mathrm{C}$. Após esse tempo o material foi resfriado a temperatura ambiente, filtrado a vácuo e lavado com uma solução de etanol e $\mathrm{HCl}$ a $2 \%$. Posteriormente o material foi calcinado a $550^{\circ} \mathrm{C}$ por 6 horas, em atmosfera de ar, para a remoção do direcionador orgânico.

\subsection{Síntese do KIT-6}


A amostra de KIT-6 foi sintetizada em condições ácidas, através do método hidrotérmico e seguindo técnica tradicional, descrita por Kleitz et al., (2003). A síntese consiste basicamente em dissolver o direcionador orgânico do tipo P123 em água e $\mathrm{HCl}$ sob agitação por 6 horas na temperatura de $35^{\circ} \mathrm{C}$. Logo após, adiciona-se o butanol e o deixa por uma hora na mesma temperatura sob agitação constante. Posteriormente adiciona-se a fonte de sílica e deixa o gel sob agitação à $35^{\circ} \mathrm{C}$ por 24 horas. Em seguida o gel é colocado na estufa para o tratamento hidrotérmico em condições estáticas e temperatura de $100^{\circ} \mathrm{C}$ por $24 \mathrm{~h}$. Logo após o material é lavado com uma solução de etanol e $\mathrm{HCl}$ à $2 \%$ e seco a temperatura ambiente. Posteriormente o material é calcinado a $550^{\circ} \mathrm{C}$ por 6 horas, em atmosfera de ar, para a remoção do direcionador orgânico.

\subsection{Caracterizações}

Para verificar se as estruturas típicas dos materiais sintetizados foram obtidas, utilizou-se a técnica de difração de raios-X. O equipamento utilizado foi um Rigaku modelo Mini Flex II utilizando radiações de $\mathrm{CuK} \alpha$, em uma voltagem de $30 \mathrm{k} / \mathrm{V}$ e corrente de tubo de $30 \mathrm{~m}$. A abertura da fenda foi de $0,15^{\circ} \mathrm{com}$ passo de $0,005^{\circ}$ e tempo de $0,4 \mathrm{~s}$, sendo os dados coletados em baixo ângulo numa faixa de $2 \theta$ de 0,5 a $3^{\circ}$.

A análise termogravimétrica foi realizada nas amostras com o intuito de se determinar a temperatura na qual as amostras seriam calcinadas. As curvas termogravimétricas foram obtidas no equipamento STA 449F3- Júpiter. Nas análises foram utilizadas aproximadamente $5 \mathrm{mg}$ de material em cadinho de porcelana, sendo as amostras submetidas a aquecimento de uma temperatura de 25 a $900{ }^{\circ} \mathrm{C}$, com uma razão de aquecimento de $10^{\circ} \mathrm{C}$.min-1, sob atmosfera dinâmica de nitrogênio com fluxo de 25 mL.min-1.

A técnica de adsorção e dessorção de nitrogênio (N2) foi realizada nas amostras a fim de se obter dados importantes sobre as propriedades texturais dos materiais sintetizados. As isotermas de nitrogênio foram realizadas em um equipamento Micromeritics ASAP 2020. As amostras foram previamente desgaseificadas, inicialmente com fluxo de nitrogênio a $90{ }^{\circ} \mathrm{C}$ por 30 min e depois no mesmo fluxo na temperatura de $200^{\circ} \mathrm{C}$ por 8 horas. Após a desgaseificação foram submetidas à adsorção de nitrogênio a $77 \mathrm{~K}$.

As amostras foram caracterizadas através da técnica de microscopia eletrônica de varredura (MEV) com o objetivo de observar morfologicamente a estrutura dos materiais sintetizados e a forma das suas partículas. Para esse fim, foi utilizado o equipamento da Shimadzo modelo MIRA3 FERG. Para a realização da análise, a amostra foi depositada sobre uma fita adesiva de carbono fixada em um portaamostra.

\section{Resultados e Discussão \\ 3.1. Difração de Raio-X (DRX)}

O Gráfico 1 mostra os resultados obtidos através da técnica de difração de raios-x realizada nas amostras de SBA-15 e KIT-6.
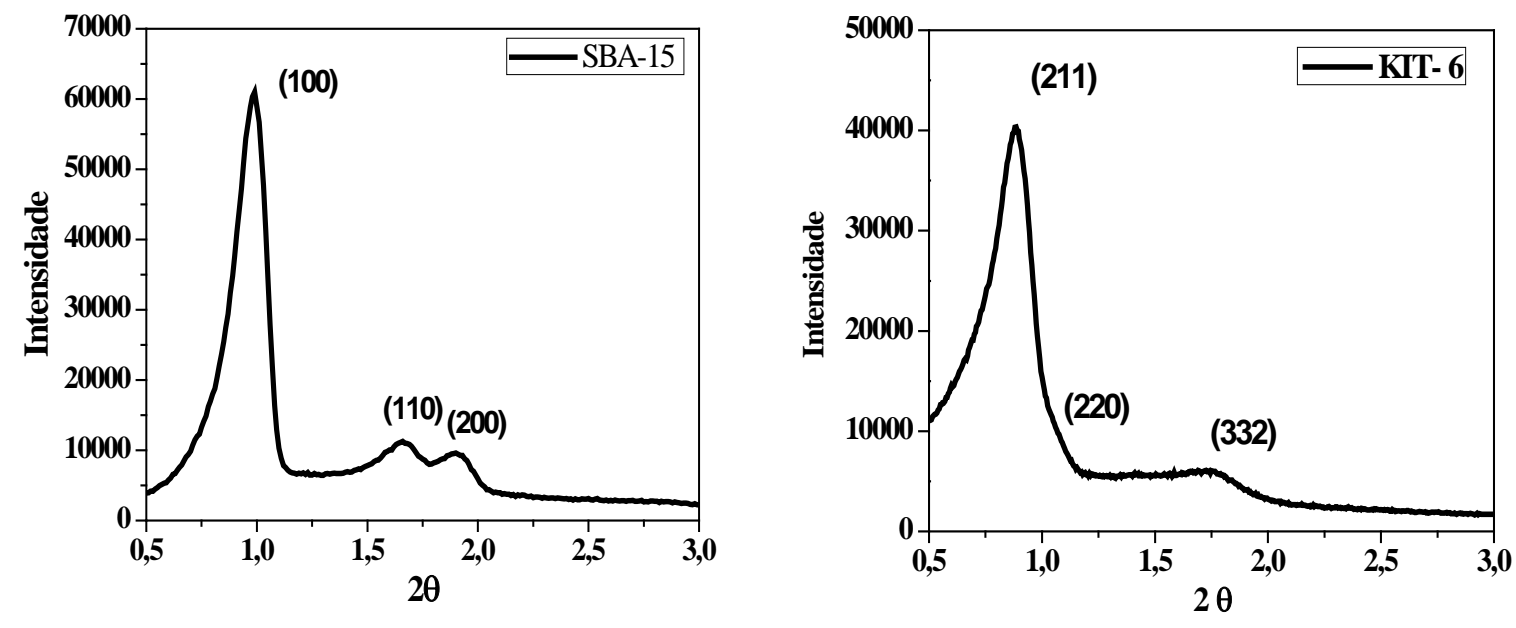
Pode-se observar que a amostra de SBA-15 apresentou três reflexões referentes aos planos de difração (100), (110) e (200), característicos da estrutura mesoporosa bidimensional com simetria P6mm, que representam, conforme a literatura, a estrutura hexagonal de materiais do tipo SBA-15 bem ordenados (ZHAO, et al. 1998 b; JING, et al. 2014). Para a amostra de KIT-6 pode- se observar três reflexões típicas, de materiais desse tipo, com índices de Miller (211), (220) e (332), sendo o primeiro pico bastante intenso, já o segundo e o terceiro são mais discretos, respectivamente. Essas reflexões representam, conforme a literatura, uma organização estrutural de grupos de silicato na forma cúbica de corpo centrado Ia3d (ZHAN et al., 2010; HE et al., 2012).

A diferença estrutural observada no DRX para as amostras pode ser explicada pelo mecanismo de formação desses materiais (Figura 1). No período inicial da síntese desses materiais há a dissolução do P123 e a formação micelar e a organização dos bastões micelares nas estruturas correspondentes. Pode- se observar que no procedimento de síntese desses materiais há uma diferença no tempo destinado a essa etapa, essa diferença de tempo está associada a diferença de estruturas, pois uma organização hexagonal 2D observada no SBA-15 teoricamente tende a se formar com mais facilidade do que uma estrutura cúbica 3D vista no KIT-6, esse fato explica o uso do Butanol como co-direcionador e cosolvente na síntese do KIT-6.

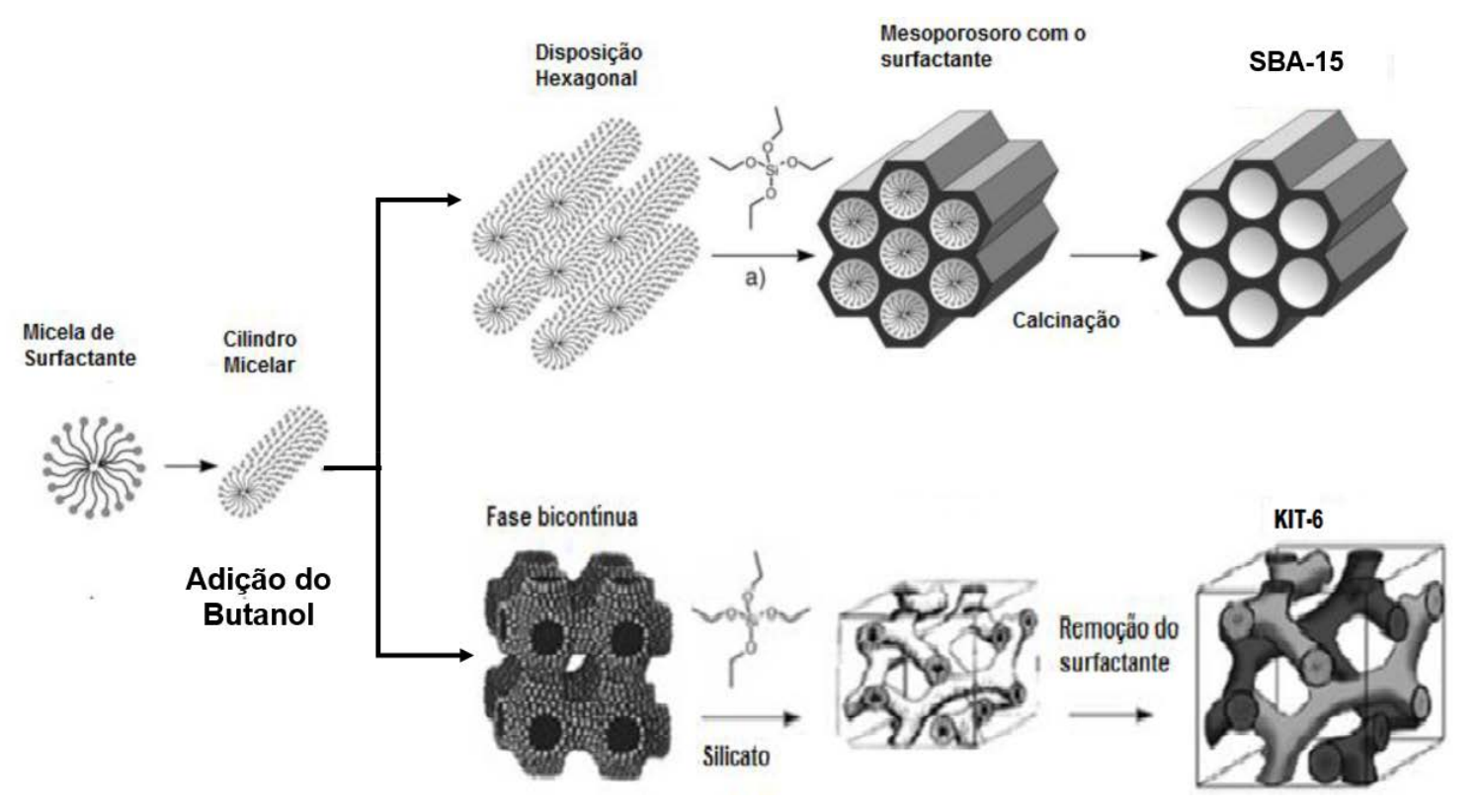

Figura 1. Esquema do mecanismo de formação dos materiais mesoporoso SBA-15 e KIT-6. Adaptado de SANTOS (2012) e OLIVEIRA (2012).

\subsection{Análise Termogravimétrica (TG/ DTG)}

O Gráfico 2 mostra os resultados obtidos através da análise termogravimétrica realizada nas amostras de SBA-15 e KIT-6. 

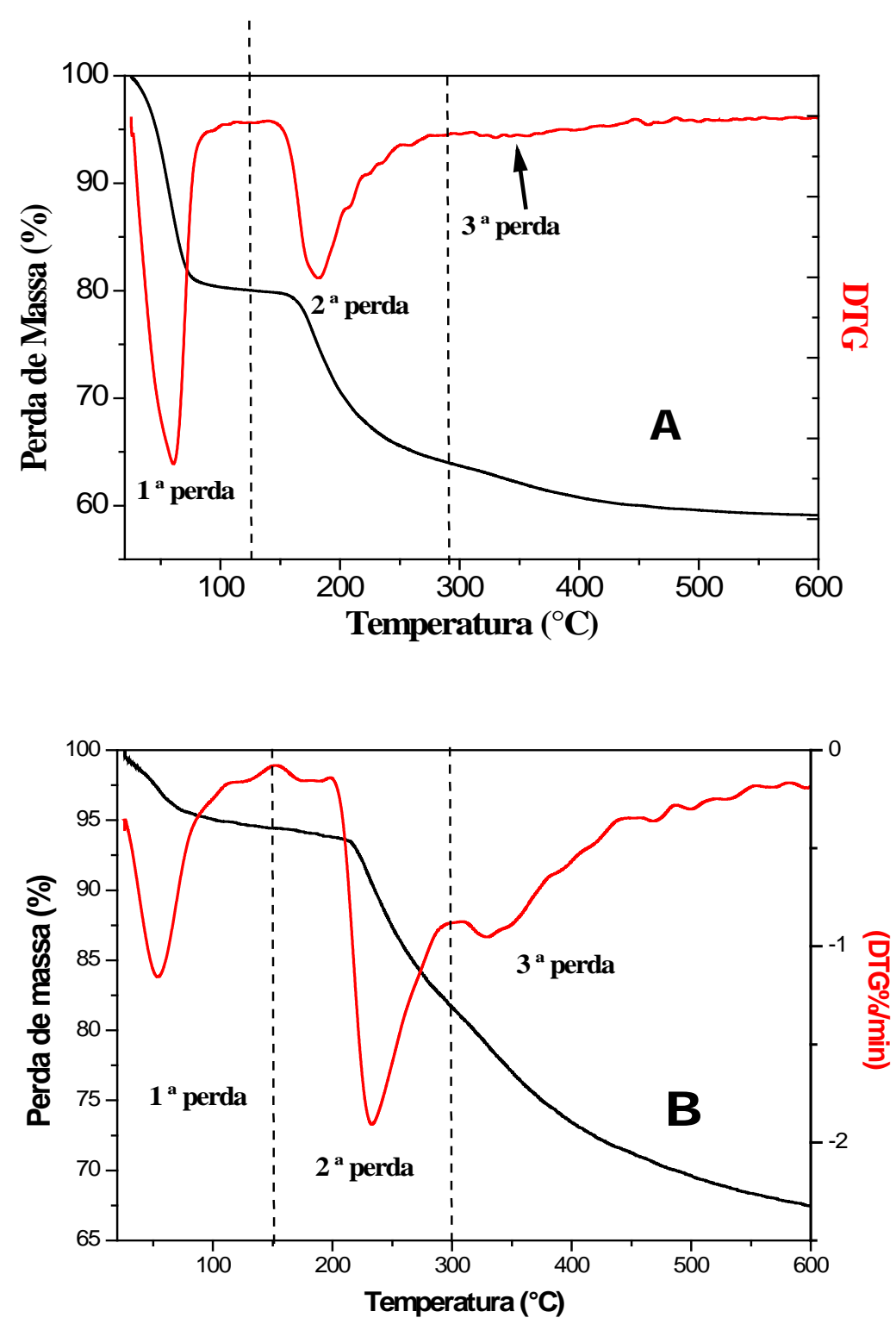

Gráfico 2. Curvas termogravimétricas das amostras a) SBA- 15 e b) KIT-6.

Pode- se observar no Gráfico 2, que as curvas obtidas, tanto para a amostra de SBA-15 quanto para a amostra de KIT-6, apresentaram três faixas de temperatura onde ocorrem perdas de massa. As três perdas correspondem a saída de água fisissorvida do material, a decomposição do direcionador orgânico e a condensação de grupos silanóis, respectivamente.

As curvas apresentaram uma diferença significativa em relação a segunda perda de massa (referente a saída do direcionador), a amostra de SBA-15 apresentou um pico mais estreito, na DTG, centrado em $190^{\circ} \mathrm{C}$ e a amostra de KIT-6 apresentou essa mesma perda, com um pico um pouco mais largo centrado em $230^{\circ} \mathrm{C}$. Essa diferença pode estar atribuída, assim como pôde ser visto no DRX, a diferença de estrutura entre materiais (Figura 1). A estrutura hexagonal com canais lineares do SBA-15, propicia uma saída mais facilitada do direcionador orgânico degradado, já a estrutura cúbica em 3D com canais bi contínuos, presentes no KIT-6, dificulta um pouco a saída desse material, aumentando assim a faixa de temperatura vista na TG. 


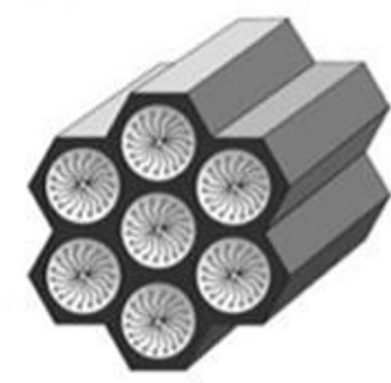

\section{Estrutura Hexagonal Estrutura cúbica}

Figura 2. Diferença de estruturas e disposição dos canais do SBA-15 (a esquerda) e do KIT-6 (a direita). Adaptado de SANTOS (2013).

\subsection{Adsorção/ Dessorção de N2}

O Gráfico 3 e a Tabela 2 apresentam dados a respeito da técnica de adsorção/dessorção de N2 realizada nas amostras de SBA-15 e KIT-6.
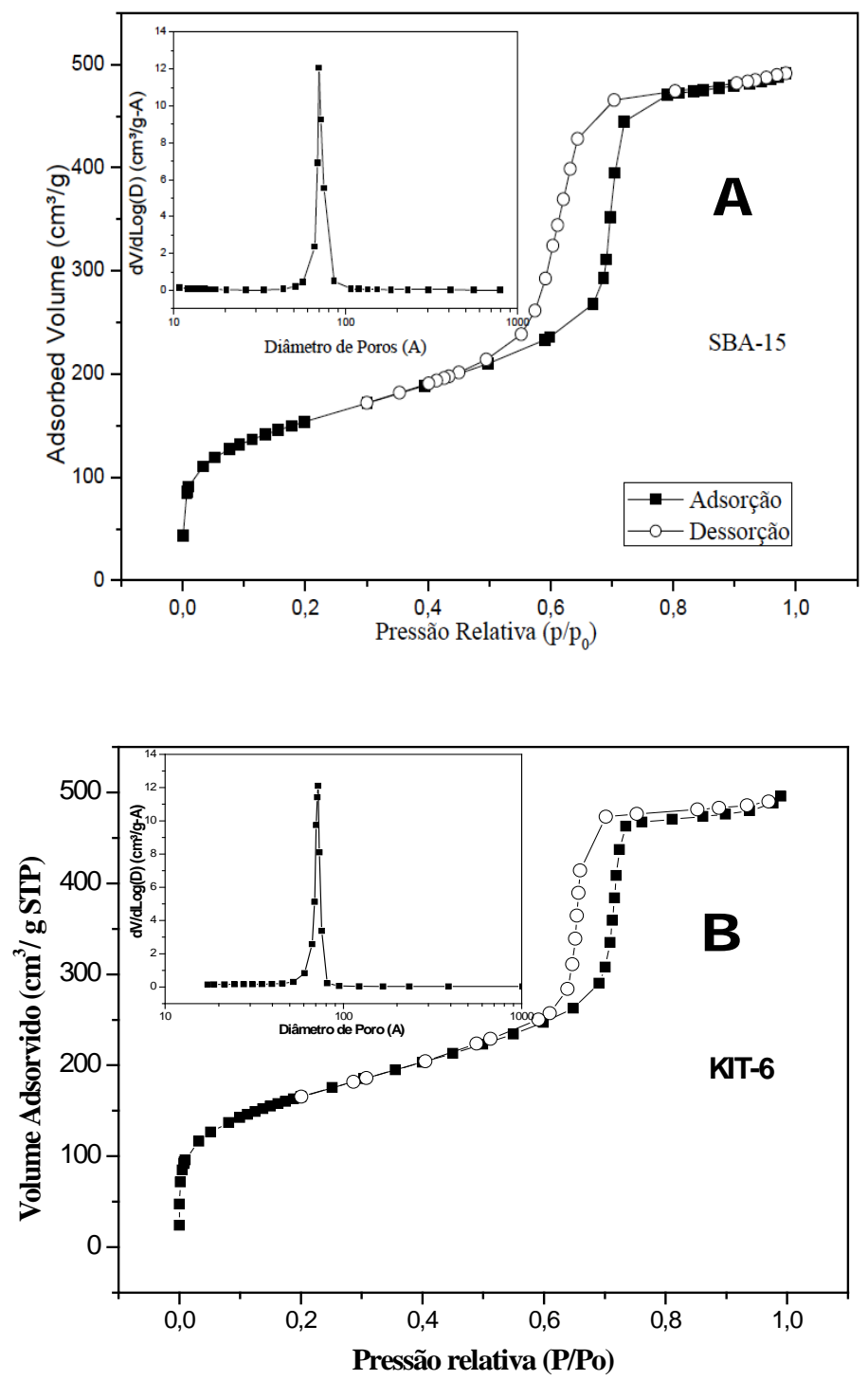

Gráfico 3. Isotermas e diâmetro de poros das amostras: a) SBA-15 e b) KIT-6.

Tabela 1. Dados das propriedades texturais das amostras SBA- 15 e KIT-6. 


\begin{tabular}{|c|c|c|c|c|c|c|c|}
\hline $\begin{array}{l}\text { Materiais } \\
\text { Mesoporos }\end{array}$ & $\begin{array}{c}\text { Dp } \\
(\mathbf{n m})\end{array}$ & $\begin{array}{c}\text { Vp } \\
\left(\mathrm{cm}^{3} \cdot \mathrm{g}^{-1}\right)\end{array}$ & $\begin{array}{c}V_{\text {Micro }} \\
\left(\mathrm{cm}^{3} \cdot \mathrm{g}^{-1}\right)\end{array}$ & $\begin{array}{c}S_{\text {BET }} \\
\left(\mathrm{m}^{2} \cdot \mathrm{g}^{-1}\right)\end{array}$ & $\begin{array}{c}S_{\text {Micro }} \\
\left(\mathrm{m}^{2} \cdot \mathrm{g}^{-1}\right)\end{array}$ & $\begin{array}{c}\mathbf{a}_{0} \\
(\mathrm{~nm})\end{array}$ & $\begin{array}{c}w \\
(\mathrm{~nm})\end{array}$ \\
\hline SBA-15 & 6,19 & 0,75 & 0,098 & 705 & 148,30 & 10,50 & 4,31 \\
\hline KIT-6 & 7,2 & 0,76 & 0,034 & 595 & 87,77 & 11,28 & 4,78 \\
\hline
\end{tabular}

Os resultados de adsorção/dessorção mostraram que ambas as amostras apresentaram isotermas do tipo IV com loop de histerese 1 em uma pressão entre 0,6 - 0,8 que é típico para materiais mesoporosos, que apresentam também microporos, altamente organizados e com tamanho de poro uniforme, referentes a esse tipo de material (HUSSAIN et al, 2013; BERNARDONI e FADEEV, 2011), esses resultados corroboram com as reflexões obtidas na técnica de DRX.

Pode- se perceber uma diferença significativa, principalmente, em relação ao diâmetro de poro, a área superficial e a microporosidade dos materiais. Essas diferenças podem estar relacionadas com o tempo de envelhecimento dos materiais na estufa. O menor tempo destinado a este fim na síntese do KIT-6 culmina em uma menor condensação de grupos silanóis (o que pode ser visto também na terceira perda de massa observada na técnica de TG). Esse fato pode explicar a menor área superficial encontrada e o leve aumento no diâmetro de poros observado. Com relação a microporosidade dos materiais, o maior tamanho dos mesoporos observado no KIT-6 pode estar ocupando espaços de possíveis microporos e sobrepondo os mesmos, o que acarreta em uma diminuição na área microporosa desse material.

\subsection{Microscopia Eletrônica de Varredura (MEV)}

A Figura 3 mostra as imagens realizadas na técnica de microscopia eletrônica de varredura, para as amostras de SBA-15 e KIT-6.
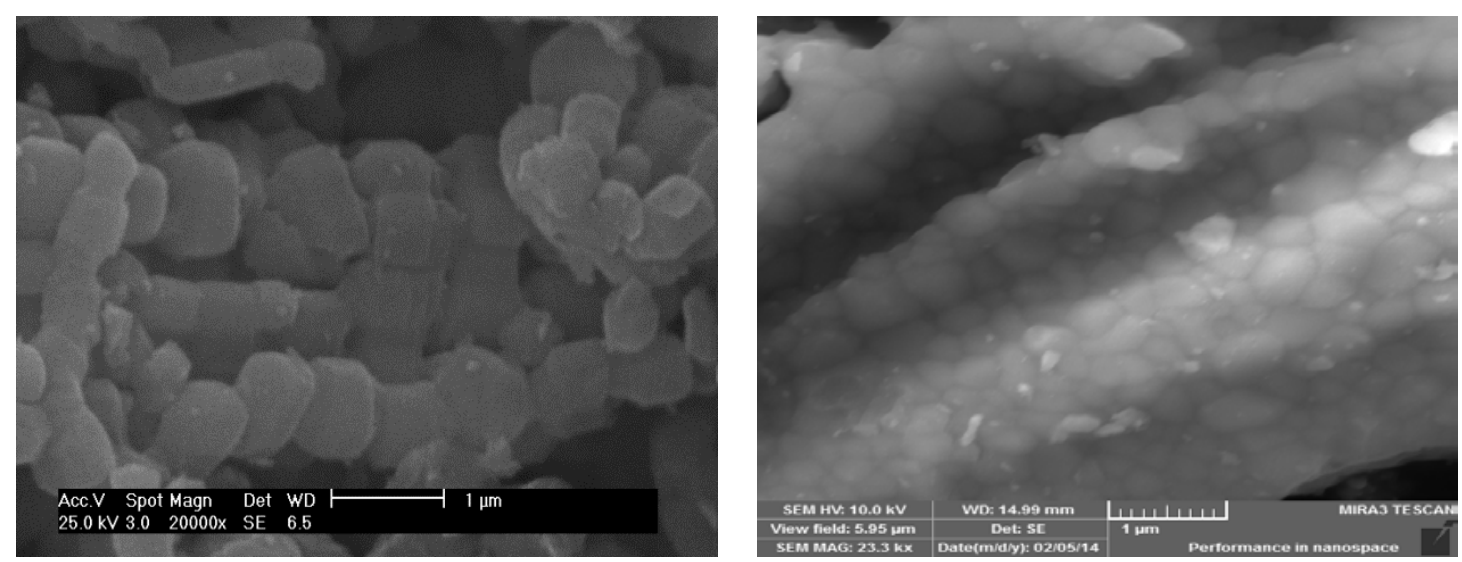

Figura 3. Imagem de MEV para as amostras de SBA-15 (a esquerda) e KIT-6 (a direita).

Pode-se observar que a amostra de SBA-15 apresentou tamanho de partículas em escala micrométrica, não uniformes e com um formato alongado, semelhantes a bastonetes, onde essas partículas se aglomeram de tal forma que o seu aspecto se assemelha a "colares de contas entrelaçados", essas estruturas condiz com as encontradas na literatura ( KATIYAR et al., 2006).

Para a amostra de KIT-6 pode- se observar que as partículas se encontram bastante organizadas. As partículas apresentam formas e tamanhos irregulares (BOULAOUED et al., 2012), na qual, os tamanhos em escala nanométrica variaram entre 100 e $500 \mathrm{~nm}$. Apesar das formas irregulares pode- se observar claramente a tendência a forma cúbica referente à estrutura característica do KIT-6. 


\section{Conclusões}

Os materiais foram sintetizados com êxito, apresentando resultados estruturais e morfológicos condizentes com os da literatura. O KIT-6 possui uma estrutura mais difícil de se formar do que a do SBA-15 e por isso se utiliza o Butanol como co-direcionador e co-solvente. As diferenças texturais entre os materiais podem ser explicadas pelo tempo de envelhecimento dos mesmos e consequentemente pela diferença na condensação dos grupos silanóis. As diferenças nos procedimentos de síntese afetam diretamente as características estruturais e morfológicas dos materiais.

\section{Synthesis of mesoporous materials: A comparative study of the SBA-15 and} KIT-6

Albstract: Ordered mesoporous materials have received much attention due to their potential applications in catalysis, adsorption, ion exchange and manufacture of advanced functional materials. Among the mesoporous materials SBA- 15 and KIT 6, which have hexagonal and cubic structures respectively, have interesting characteristics such as high thermal and hydrothermal stability, well ordered pore systems, high surface area and high volume and diameter Average pore. The synthesis of these materials hydrothermal occurs in acidic medium, using P123 as structure director and TEOS as silica source, and the synthesis of KIT-6, different from SBA-15, also occurs the addition of butanol as a co- solvent and co-driver. The objective of this study is to synthesize and characterize the mesoporous materials SBA- 15 and KIT- 6 in order to carry out a comparative study of the materials, assessing structural and morphological aspects of them. The materials were prepared by procedures found in the literature and characterized by means of XRD techniques, TG / DTG, adsorption / desorption of N2 and SEM. The materials were successfully synthesized, having typical characteristics. It might be observed by characterization of the main structural and morphological differences between the two types of materials. The SBA-15 has a hexagonal structure easier to form compared to KIT-6 cubic structure. Need to the addition of the butanol in the initial part of the KIT- 6 synthesis, for the organization of the micelles in the cubic form.

Keywordls: Synthesis; Characterization; SBA-15; KIT-6.

\section{Referências bibliográficas}

BOULAOUED, A. et al. Mo/KIT-6, Fe/KIT- 6 and Mo-Fe/KIT- 6 as new types of heterogeneous catalysts for the conversion of MCP. Microporous and Mesoporous Materials. v. 155, p. 131-142, 2012.

DAO, L.; GUOPI NG, C.; XINGYI, W. Incorporation of lanthanum into SBA-15 and its catalytic activity in trichloroethylene combustion. J ournal of Rare Earth, v. 26, p. 717-721, 2008.

DOU, B. et al. Adsorption performance of VOCs in ordered mesoporous silicas with different pore structures and surface chemistry. J oumal of Hazardous Materials, v. 186, p. 1615-1624, 2011.

FALAHATI, M. et al. Aminopropyl-functionalized cubic la3d mesoporous silica nanoparticle as an efficient support for immobilization of superoxide dismutase. Biochimica et Biophysica

USSAIN, M. et al. Novel Ti-KIT- 6 material for the photocatalytic reduction of carbon dioxide to methane. Catalysis Communications, v.36, p.58-62, 2013.

KALBASI, R. J.; MOSADDEGH, N. Pd-poly(N-vinyl-2-pyrrolidone)/KIT-6 nanocomposite: Preparation, structural study, and cataActa, v. 1814, p. 1195-1202, 2011.

Hlytic activity. C. R. Chimie. v.15, p.988-995, 2012. 
KARTHIKEYAN, G.; PANDURANGAN, A. Post synthesis alumination of KIT-6 materials with Ia3d symmetry and their catalytic efficiency towards multicomponent synthesis of $1 \mathrm{H}$-pyrazolo [1,2-] phthalazine-5,10-dione carbonitriles and carboxylates. J ournal of Molecular Catalysis A: Chemical, v. 361- 362, p. 58- 67, 2012.

KO, Y. G.; SHIN, S. S.; CHOI, U. S. Primary, secondary, and tertiary amines for $\mathrm{CO} 2$ capture: Designing for mesoporous CO2 adsorbents. J ournal of Colloid and I nterface Science, v. 361 p. 594-602, 2011.

KUMARESAN, L. et al. Mesoporous Ti-KIT- 6 molecular sieves: Their catalytic activity in the epoxidation of cyclohexene. J ournal of the Taiwan Institute of Chemical Engineers. v.41, p.670-675, 2010.

NAKAZAWA, J. et al. Discrete Complexes I mmobilized onto Click-SBA-15 Silica: Controllable Loadings and the Impact of Surface Coverage on Catalysis. J. Am. Chem. Soc., v.134 (5), p.2750-2759, 2012.

PRABHU, A. et al. Synthesis and characterization of aluminium incorporated mesoporous KIT-6: Efficient catalyst for acylation of phenol. Applied Catalysis A: General, v. 360, p. 59-65, 2009.

QIAN, L. et al. Influence of KIT-6's pore structure on its surface properties evaluated by inverse gas chromatography. Chemical Engineering J ournal. v. 213, p. 186-194, 2012.

SANTOS, A. P. B. Síntese, caracterização e estudo cinético da degradação de quitosana impregnada em SBA-15. 2012. 90 f. Dissertação (Mestrado em Química) Universidade Federal do Rio Grande do Norte, Natal, RN, 2012.

SONI, K. et al. 3-D ordered mesoporous KIT-6 support for effective hydrodesulfurization catalysts. Applied Catalysis B: Environmental, v.90, p.55-63, 2009.

WANG, W. Q. et al. Effect of alcohol on morphology and mesostructure control of anionic-surfactanttemplated mesoporous silica (AMS). J ournal of Colloid and I nterface Science. v. 331, p. 156162, 2009.

ZHAN, W. et al. Synthesis of lathanum or La-B doped KIT-6 mesoporous materials and their application in the catalytic oxidation of styrene. J ournal of rare earths, v.28, p. 369-375, 2010.

ZHAO, D. et al. Triblock Copolymer Syntheses of Mesoporous Silica with Periodic 50 to 300 Angstrom Pores. Science. v, 279, p. 548-552, 1998b. 